\title{
A MOBILE APPLICATION FOR VIRTUAL HERITAGE AND UGC PUBLIC SHARING
}

\author{
Li Gongli $^{\mathrm{a}}$, Shang Jin ${ }^{\mathrm{b}, *}$, Chen Huilian ${ }^{\mathrm{a}}$
}

a Beijing Qingda Transsim Technologies, Inc. 100085 Beijing, China - ligongli@163.com; huilian.chen@foxmail.com

${ }^{\mathrm{b}}$ Dept. of Architecture and Urban Heritage, THUPDI, 100085 Beijing, China - shaangjin@gmail.com

KEY WORDS: Re-yuanmingyuan, Virtual Heritage, Mobile Application, UGC, LBS

\begin{abstract}
:
Heritage documentation and representation is now a growing concern in the contemporary world, with unprecedentedly rapid technological development that pushes the frontier further every day. This ever growing means benefits both professionals and the general public, and the two can now be connected by this virtual bridge that channels heritage information from one end of the spectrum to the other, thus facilitating a dialogue never considered before. 4D virtual heritage with visualized tempo-spatial information can be easily shared across the continents and the story of heritage is told by a simple move of the thumb. Mobile LBS (Location-Based Service) enhances visitors' on-site experience and is readily available on the popular iOS platform. UGC (User Generated Content) on the internet provides interaction among users and managers, and brings the heritage site and the public into a live conversation. Although the above technological exploration is promising in itself, the question still remains as how it may be best implemented. The Re-yuangmingyuan program for the reconstruction and representation of an imperial garden in Beijing has made several attempts that deserve discussion, and contributes to heritage documentation and conservation in general.
\end{abstract}

\section{INTRODUCTION}

As cultural heritage is attracting more attention from all sectors around the globe, there is an increasing need to explore the technological potential at the service of heritage documentation and interpretation. In the attempts to digitally document perished built heritage and landscape and to generate virtual reconstructions, a methodology has been gradually developed that can be applied to East Asian timer structures (He Yan, 2012). With an experienced team and mature process, similar archaeological sites are almost ready to come back to life again. However, the next question is how to present the reconstruction to the audience as effective as possible? The following are the technological answers, as demonstrated in the Reyuanmingyuan program.

\section{MOBILE APPLICATION FOR VIRTUAL HERITAGE}

\subsection{Technological Background}

As new technology continues to emerge, virtual heritage, as an effective means for cultural heritage presentation in the modern technological context, gradually becomes a major field in heritage research, conservation, and interpretation (Karina et al., 2012). Digital research and reconstruction of heritage facilitates a complete archive with tempo-spatial information and various cultural content, to be presented in a comprehensive 4D output.

The foundation of a mobile representation platform is the mature heritage data. The internet and mobile device, characterized by instant connection, provides immediate update and sharing of the content. Technologies like IOT (Internet Of Things), GPS positioning, QR (Quick Response) code may generate a dynamic link between the real and virtual world. (Figure 1)

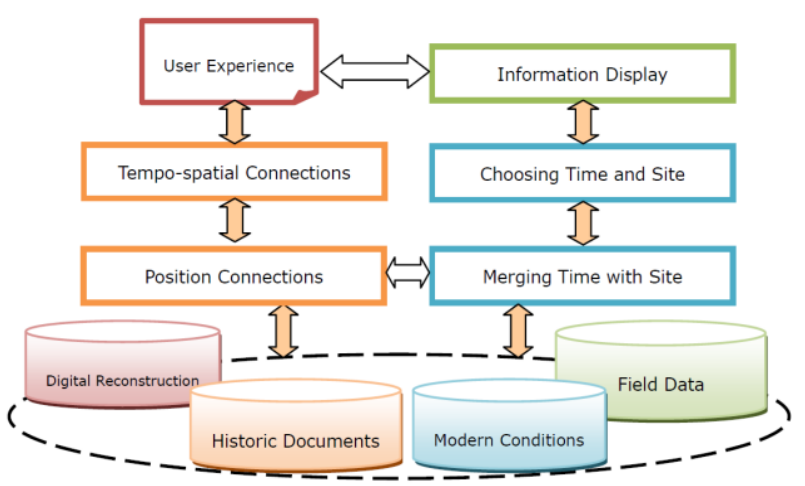

Figure 1. Framework of a Mobile Representation Platform

\subsection{The Virtual Heritage of Yuanmingyuan}

The Re-yuanmingyuan mobile application, based on the scientific documentation and reconstruction, realizes a virtual tour on the mobile iOS platform for the imperial garden of Yuanmingyuan from 1707 to 1860 (He Yan et al., 2011). Visitors will first see the master plan with pre-defined route in red. (Figure 2) Before entering, they can look at the site information and choose an optimum travel route that includes all the interesting scenic spots to visit. Once on their way, GPS will guide visitors by showing the real time location with a red dot on the route of the intelligent map. Virtual reconstruction and the physical location is therefore integrated to facilitate smart heritage tour.

\footnotetext{
* Corresponding author.
} 


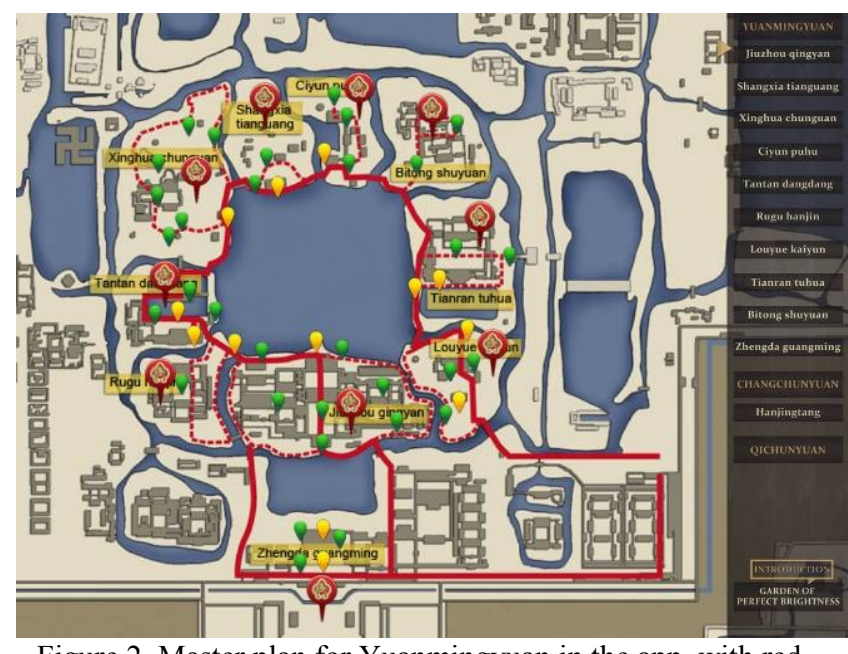

Figure 2. Master plan for Yuanmingyuan in the app, with red lines showing pre-defined visitor route

\section{MOBILE APPLICAITON AND LBS}

\subsection{Theoretical Consideration}

Mobile application features a close connection with the physical position. Its public-oriented map output and LBS technology combines the virtual heritage with the real environment, while geographical positioning enhances visitor's actual experience in the on-site tour. Based on positioning information and various IOT technology, on-site spots can trigger the heritage information presentation and communication point. This will enrich both the amount of heritage information and the form of interpretation. A further location-based combination of heritage information at all tempo-spatial points will generate a tour route. Visitors at the site can comment in the application at specific spots, contributing to the mobile platform-based 'concurrence' of heritage documentation.

\subsection{LBS Application in Yuanmingyuan}

One way to implement to LBS service in the site is to erect QR code stands to notify visitors spots of interest (Fig. 4). The iOS app, on reading the code, will bring up the heritage information in the program, including reconstruction images and audio interpretation. This audiovisual information will enhance visitors' on-site experience, particularly important for sites like Yuanmingyuan, where little built heritage remains.

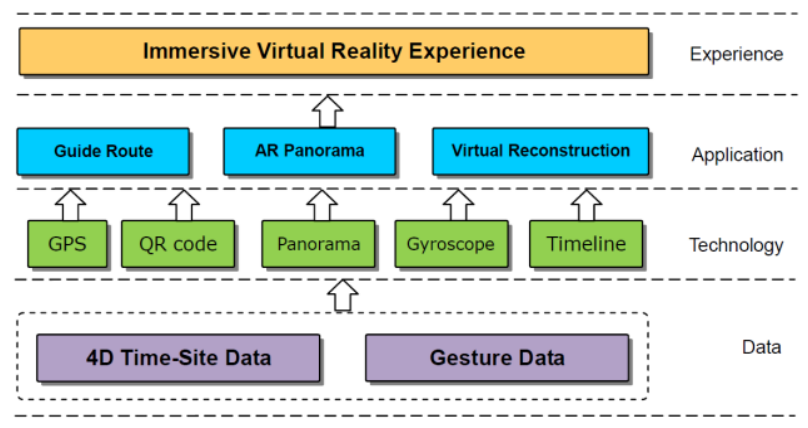

Figure 3. VR Generation Structure and Strata

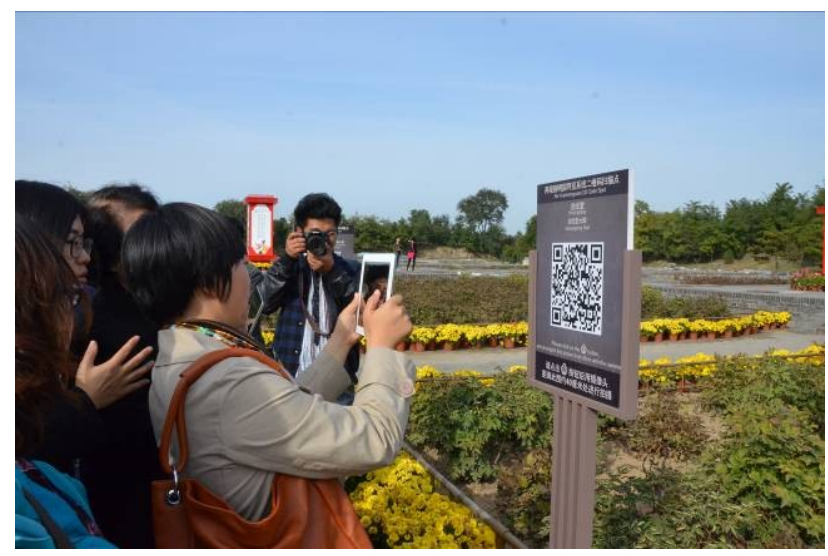

Figure 4. Visitors reading the QR code with an iPad

Beside reconstruction renderings, the app also features a panorama view which may also be triggered by reading the QR code. This allows user to emerge oneself in a 360-degree virtual environment that is able to present the 3D reconstruction from every possible angle. (Figure 5) The embedded gyroscope captures user's gesture data and combines it with simultaneous movement in virtual scenes, which generates realistic experience. Although more informative and impressive than a single rendering, the panorama is larger in size and requires better hardware support. Therefore only the most impressive sites are chosen for this feature.

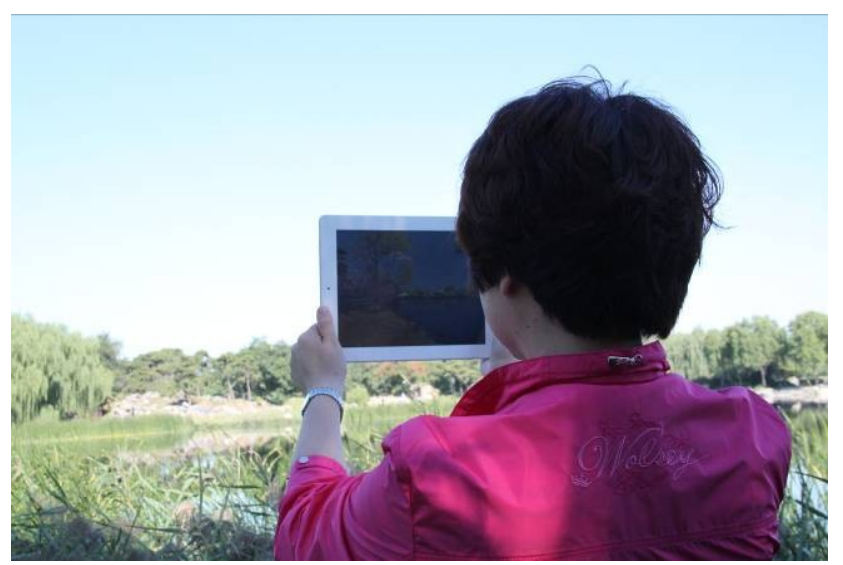

Figure 5. Visitors looking at AR reconstruction in an iPad

\subsection{D Reconstruction and the Time Wheel}

The historic development of heritage is also an important aspect in representation (T.P. Kersten et al., 2012). With multiple forms of data, virtual heritage at different time and places can be accessed on the mobile platform. Research on Yuanmingyuan has shown that most structures have been transformed several times during each reign of the emperors. Period-based reconstructions are incorporated in the rendered scenes of each scenic area, and the viewpoints displayed by a red arrow. (Figure 6) 


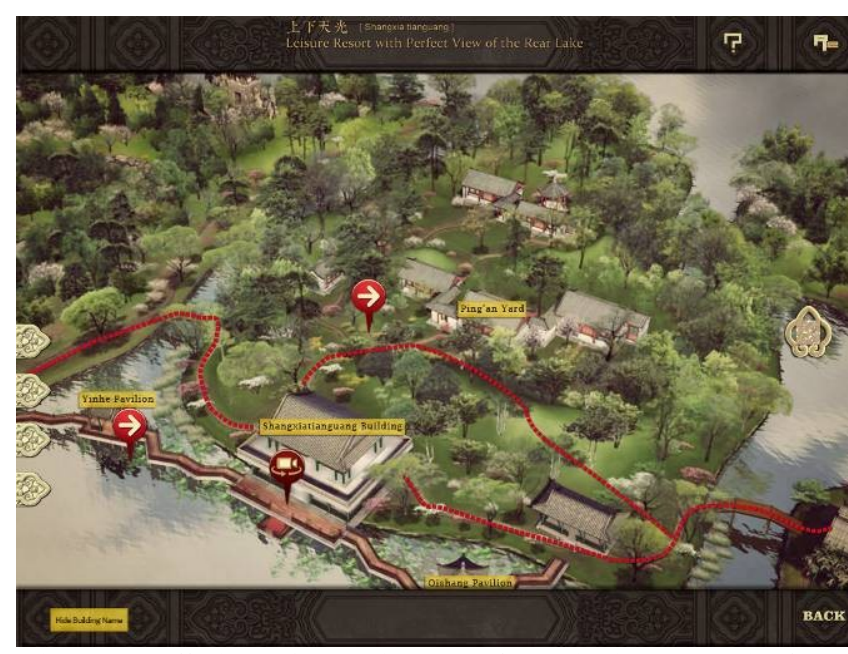

Figure 6. Full view of a scenic area (Shangxia tianguang) in the app, with red arrows showing view points of rendered images

For a specific site, reconstructions of the building in successive periods are rendered and organized in a specially designed diachronic Time Wheel. (Figures 7 to 9) By turning the Wheel that shows each transformation, comparisons can be made at the same location to further visitors' understanding of the site. The last rendering on the petal of the Wheel is actually a documentation of the site during modern excavations. In this manner, visitor and professional alike can learn from this special feature.

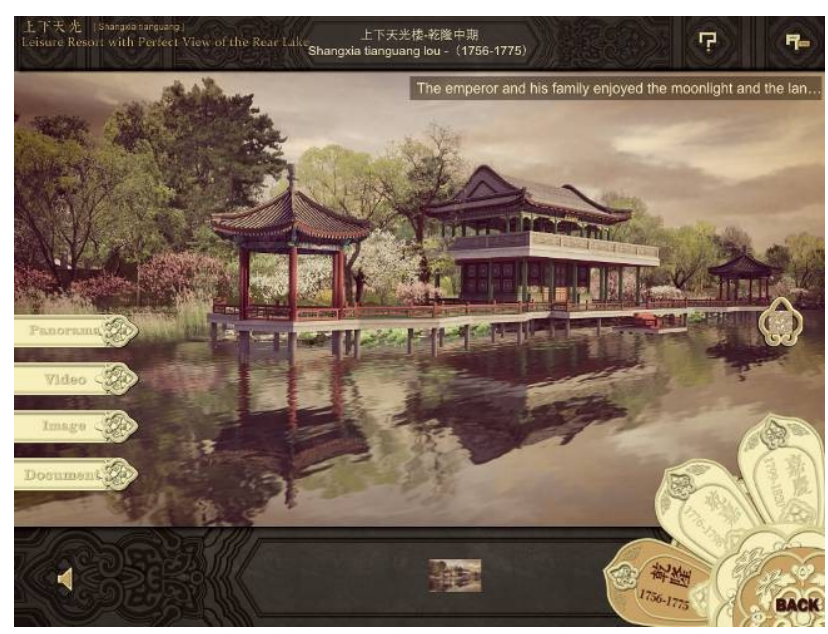

Figure 7. Rendered image of a corresponding site (Shangxia tianguang), showing an earlier state the monument (1756-1775)

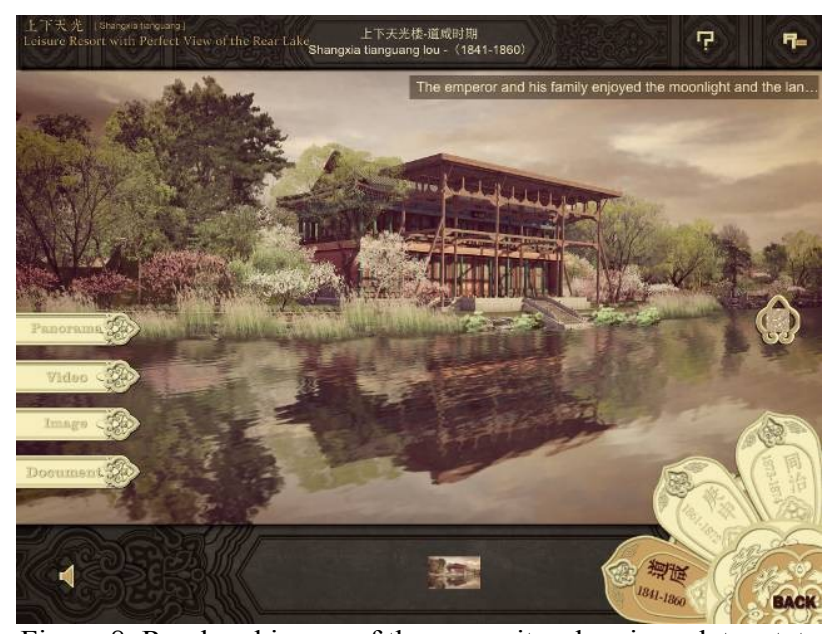

Figure 8 . Rendered image of the same site, showing a later state the monument (1841-1860)

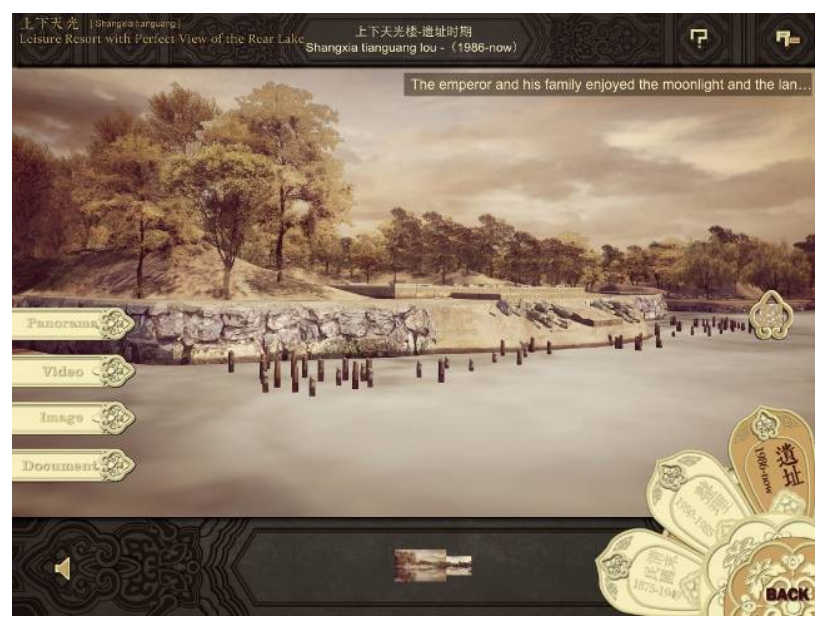

Figure 9. Rendered image of the same site, as excavated (1986)

\section{THE FUTURE - UGC AND CROSS-PLATFORM PUBLIC SHARING}

One of the major bottlenecks for virtual heritage is a lack in available channel for public sharing. Based on virtual panorama and on-site mobile information interactions, attempts are made to use modern mobile UGC technology to channel multidimensional information towards visitors.

Advanced web technology will be fully utilized to attract and organize public participation. Microblog, SNS, web forum, video and images are integrated to enhance public experience from traditional web browsing, and facilitate content generation and communication for wider sharing and engagement.

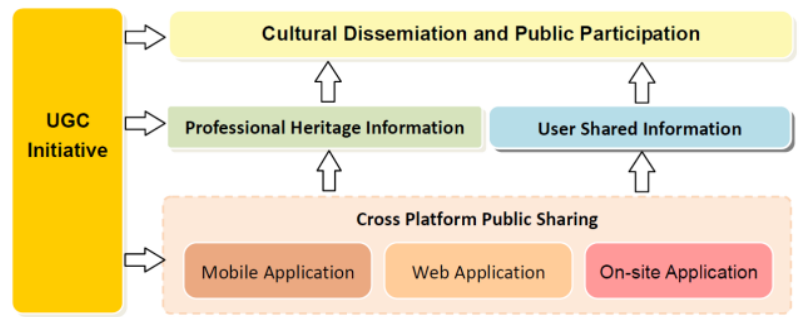

Figure 10. UGC and Public Sharing 
In the ear of Web 2.0, most content in the network comes from users. Each person may contribute on an individual basis, and the internet content is not generated by a single party as before. This simply takes the explosion of information one step further, with a more comprehensive perspective, more specialized areas and much richer content. Human knowledge accumulates and benefits from such technological advancement in an unprecedented way.

Originated from the internet, UGC developed with the rise Web 2.0 that allowed users to upload self-made content to be shared across other users. Characterized by individuation, UGS is not a specific service, rather a new way people use the internet, which shifts the pattern from the previous one-way download to twoway upload/download. An integration of Ajax and Web 2.0 with digital map will provide interactive tour information on the internet. Heritage manager and fans may also contribute to information gathering, data processing and publishing. The establishment of an interactive information platform casts new light on the innovation of intelligent heritage conservation and communication. Therefore, with features like built-in microblog portal, the user group will be significantly magnified and thus greater sharing among the public.

\section{CONCLUDING REMARKS}

Since its release in 2012, the Re-yuanmingyuan app has gained extensive attention from both the academia and public, and ranked the top of download for days. QR code stands in the park worked well with the app, which facilitated an unprecedented experience as far as historic parks are concerned in China. The theoretical proposals of a UGC oriented interactive heritage information application have been well tested in practice. Although among the first examples of such attempts, there is still room for improvement. Interactive design can be made more friendly, e. g. smoother visual transition when images change at each turn of the Time Wheel. The content can be organized in a more attractive manner as though telling stories. A smaller size of the app will also make it easier to launch. It is true that, at the very nexus of public participation, more immediate interaction will combine technology with heritage, and yield ever better products for heritage representation and interpretation.

\section{References from Books:}

For a detailed methodology and explanation of the reconstruction process of Yuanmingyuan scenic areas, see Guo Daiheng, He Yan, 2012. A Digital Reconstruction of Yuanmingyuan, Zhongxi Book Company, Shanghai.

\section{References from other literature}

He Yan, 2011. Re-relic/ Yuanmingyuan: an effective practice in virtual restoration and visual representation of Cultural Heritage. In: XXIII CIPA Symposium 2011 Proceedings, Prague, Czech Republic

(http://cipa.icomos.org/fileadmin/template/doc/PRAGUE/071.p df)

He Yan, et al., 2012. A Virtual Reconstruction Methodology for Archaeological Heritage in East Asia - Practical Experience from the Re-relic Program in China. In: $4^{\text {th }}$ International Meeting on Graphic Archaeology and Informatics, Cultural Heritage and Innovation Proceedings, Seville, Spain. (Past proceedings should be available at http://www.arqueologiavirtual.com/arqueo/)
Karina Rodriguez Echavarria, Jaime Kaminski, and David Arnold, 2012. 3D Heritage on Mobile Devices: Scenarios and Opportunities. In: 4th International Conference, EuroMed 2012 Proceedings, Limassol, Cyprus. pp.149-158

Thomas P. Kersten, et al., 2012. Automated Generation of an Historic 4D City Model of Hamburg and Its Visualisation with the GE Engine. In: 4th International Conference, EuroMed 2012 Proceedings, Limassol, Cyprus. pp.55-65

\section{Acknowledgements}

Professor Guo Daiheng from Tsinghua University deserves our gratitude in guiding us with the historic research on Yuanmingyuan throughout the Re-relic program. The authors would like to thank He Yan for establishing the foundation and guiding principles of the program and all the efforts of the team. 